\title{
Patient characteristics and procedural outcomes of moderate sedation for endoscopic procedures in patients with obesity: A retrospective, propensity score-matched study
}

\section{(ㄷ)(1) $(-)$}

Authors

Shashank Garg ${ }^{1}$, Shorabuddin Syed ${ }^{2}$, Abhilash Perisetti ${ }^{1}$, Sumant Inamdar ${ }^{1}$, John Vargo $^{3}$

Institutions

1 Division of Gastroenterology and Hepatology, Department of Medicine, UAMS, Little Rock, Arkansas, United States

2 Department of Biomedical Informatics, University of Arkansas for Medical Sciences, Little Rock, Arkansas, United States

3 Department of Gastroenterology, Hepatology and Nutrition, Digestive Disease and Surgery Institute, Cleveland Clinic, Cleveland, Ohio, United States

submitted 10.3.2021

accepted after revision 13.7.2021

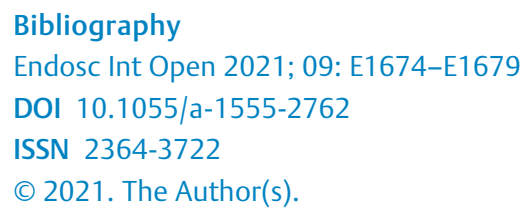

Supplementary material is available under https://doi.org/10.1055/a-1555-2762

\section{ABSTRACT}

Background Endoscopic procedures are performed commonly with moderate sedation. Obesity can pose a challenge in its safe administration. This study was aimed at assessing outcomes of endoscopy procedures performed with moderate sedation in obese patients.

Patients and methods This was a retrospective study of patients undergoing esophagogastroduodenoscopy (EGD) and/or colonoscopy with moderate sedation from July 17 , 2017 to December 31, 2019. Demographics, comorbidities, outpatient medications and procedure-related outcomes (procedure time, recovery time, cardiopulmonary adverse events, 7-day post-procedure hospitalization, cecal intubation time, withdrawal time, tolerance of moderate sedation and sedation medications administered) were compared for patient with and without obesity after propensity score matching. Standard statistical methods were used for analysis.

Results A total of 7601 procedures were performed with moderate sedation for 5746 patients. Propensity score matching identified 1360 and 1740 pairs of EGDs and colonoscopies with moderate sedation for patients with and without obesity. Recovery time was found to be shorter for obese patients undergoing EGD (OR: 0.989, $95 \% \mathrm{Cl}$ : 0.981-.998; $P=0.01)$. Obese patients did not differ from non-obese patients in any other procedure-related outcomes for EGDs or colonoscopies.

Conclusions Outcomes for endoscopy procedures performed with moderate sedation were noted to be similar between obese and non-obese patients. These findings suggest that moderate sedation can be used safely for endoscopic procedures in patients with obesity.

\section{Introduction}

About 17 to 20 million endoscopy procedures including esophagogastroduodenoscopy (EGD), flexible sigmoidoscopy and colonoscopy are performed in the United States annually [1, 2]. Majority of these procedures require some form of sedation to reduce patient anxiety, minimize patient discomfort and maximize patient safety thus allowing the endoscopist to perform a thorough examination [3]. Traditionally, moderate sedation has been used to perform these procedures. It is a pharmacologic induced state in that the patient is still conscious but is able to tolerate unpleasant stimuli and maintain cardiovascular 
status [4]. Even though moderate sedation is safe, it is associated with minor or major adverse events (AEs) in 1 per 170 to 1 per 10,000 endoscopy procedures and cardiorespiratory events make up for majority of these AEs [5]. Therefore, patients are continuously monitored during moderate sedation to avoid excessive sedation and cardiorespiratory depression. Obesity is one of the factors that make administration of moderate sedation challenging. It can affect the metabolism of most drugs used in moderate sedation altering the dose required to achieve effective sedation [6]. Obesity is typically associated with various cardiopulmonary comorbidities that may increase the rate of cardiorespiratory AEs during the procedure [7-10]. However, to date there is very limited literature on the outcomes of obese patients undergoing endoscopy procedures with moderate sedation. The aim of this study was to evaluate the outcomes of general endoscopy procedures performed with moderate sedation in patients with obesity.

\section{Patients and methods}

This was an Institutional Review Board (IRB) approved retrospective study that included all patients undergoing EGD or colonoscopy at University of Arkansas for Medical Sciences (UAMS) from July 1, 2017 to December 31, 2019. All adults (>18 years of age) who had EGD and/ or colonoscopy with moderate sedation at UAMS during the study period were included. Patients who had: 1) an EGD or colonoscopy performed with the involvement of an anesthesia provider; 2) EGD or colonoscopy without sedation; 3 ) procedures other than EGD or colonoscopy; 4) colonoscopies with documented poor bowel preparation (Supplementary file $\mathbf{1}$ ); and 5) colonoscopies that were performed together with an EGD were excluded from the study. The rationale for last criteria was that these patients had already had sedation for the EGD and therefore, we were not able to correctly assess the sedative medications administered for these colonoscopies.

End points for patients undergoing EGD were 1) total procedure time; 2 ) recovery time, 3 ) immediate cardiopulmonary AE; 4) 7-day all-cause hospitalization after the procedure, 5) poor tolerance of moderate sedation; 6) doses of medications used for the procedure; and 7) use of adjunctive sedatives for sedation during the procedure. For patients undergoing colonoscopies cecal intubation time (CIT) and withdrawal time were considered as end points in lieu of total procedure time.

Moderate sedation was defined as sedation directed by the physician performing the endoscopic procedure and provided by a nurse during the procedure using Midazolam and Fentanyl. Poor bowel preparation was defined as clear documentation of bowel prep as being "poor" or rated as $<6$ on Boston Bowel Prep Score. Adjunctive sedatives were medications that are not primarily used for sedation but have strong sedative effect and are used in conjunction with Midazolam and/ or Fentanyl i. e. diphenhydramine and prochlorperazine. Total procedure time was the time from scope insertion to complete withdrawal of the scope. Cecal intubation time was time from insertion of the colonoscope to the time cecum was reached. Withdrawal time for colonoscopies was the time from cecal intubation time to complete withdrawal of the colonoscope. Recovery time was the time patient spent in the postoperative section of the endoscopy unit before being discharged or sent back to floor. Immediate cardiopulmonary AEs were cardiac or pulmonary AEs that were noted during or immediately after the procedure and were documented in the chart including hypotension and hypoxia. Poor tolerance of moderate sedation was assessed based on the documentation in the endoscopy report. This documentation included clear documentation of tolerance of procedure, reordering the procedure with anesthesia or requirement of anesthesia for future procedures. Repeat procedures with anesthesia for therapeutic purposes was not counted as poor tolerance of the procedure.

Patients undergoing EGD or colonoscopy procedures were identified with the most commonly used administrative codes associated with both types of procedures (Supplementary file 1). Body mass index (BMI) recorded at the time of the procedure. Information about demographics (age, gender, race), history of smoking or alcohol use and provider performing the procedure (gastroenterologist, hepatologist, other) was obtained on each patient. Other provider category included surgeons and family medicine physicians. These providers had performed very few (15) EGDs in total for non-obese and obese patents. Therefore, these procedures were excluded from the analysis. All the providers had been in full time practice for $\geq 2$ years and performed $\geq 250$ colonoscopies per year. Information on all primary and secondary end points was collected. Co-morbid illness assessment was done by calculating the Elixhauser Comorbidity Index (ECI) score using the Van Walraven algorithm [11-14]. Obstructive sleep apnea (OSA) was recorded as a separate comorbidity because it is not included in $\mathrm{ECl}$ and is an important factor to consider during sedation. Number of prescription medications listed in each patient's medication list that can affect tolerance or efficacy of moderate sedation were also recorded (Supplementary file 1). Patients were divided into three groups: 0,1 or $\geq 2$ prescription medications with sedative effects. Details of pre-procedure assessment and administration of moderate sedation are provided in

\section{Supplementary file $\mathbf{1}$.}

Descriptive statistics were used to perform exploratory analyses. Categorical data were described as proportions and analyzed using chi-square test or Fisher exact test. Continuous data were described as mean (standard deviation or SD) or median (interquartile range [IQR]) and analyzed using t-test, ANOVA, Wilcoxon rank-sum test or Kruskal-Wallis test depending on the distribution of the variable. Data were analyzed separately for EGDs and colonoscopies performed with moderate sedation. Some patients underwent more than one EGD or colonoscopy. Therefore, per-procedure analysis was conducted. Perpatient sensitivity analysis was conducted to determine if repeat procedures for the same patient were affecting the study results. Exploratory analysis using standard statistical methods showed that the obese and non-obese groups were dissimilar to each other age, gender, race, number of prescribed medications with sedative effects, Elixhauser comorbidity index score, OSA and tobacco and alcohol use. Therefore, propensity score matching (PSM) was performed to make the study groups com- 
- Table 1 Demographics and clinical characteristics of patients with and without obesity undergoing EGD and colonoscopy with moderate sedation after propensity score matching.

\begin{tabular}{|c|c|c|c|c|c|c|}
\hline & \multicolumn{3}{|l|}{ EGD } & \multicolumn{3}{|l|}{ Colonoscopy } \\
\hline & $\begin{array}{l}\text { Non-obese } \\
(\mathrm{BMI}<30 \text {; } \\
\mathrm{n}=1360 \text { ) }\end{array}$ & $\begin{array}{l}\text { Obese } \\
(B M I \geq 30 \\
n=1360)\end{array}$ & $P$ value & $\begin{array}{l}\text { Non-obese } \\
(\mathrm{BMI}<30 \text {; } \\
\mathrm{n}=1740 \text { ) }\end{array}$ & $\begin{array}{l}\text { Obese } \\
(B M I \geq 30 \\
n=1740)\end{array}$ & $P$ value \\
\hline Mean age in years (SD) & $57.12(16.02)$ & $55.68(13.47)$ & 0.01 & $57.46(13.74)$ & $57.21(12.04)$ & 0.56 \\
\hline Female (\%) & $839(61.69)$ & $886(65.15)$ & 0.06 & 1049 (60.29) & $1161(66.72)$ & $<0.0001$ \\
\hline Race (\%) & & & 0.88 & & & $<0.001$ \\
\hline - White & $936(68.82)$ & $929(68.31)$ & & $1104(63.45)$ & $977(56.15)$ & \\
\hline - African-American & $368(27.06)$ & $370(27.21)$ & & $558(32.07)$ & $713(40.98)$ & \\
\hline - Other & $56(4.12)$ & $61(4.49)$ & & $78(4.48)$ & $50(2.87)$ & \\
\hline Prescribed medications with sedative effects (\%) & & & 0.06 & & & $<0.01$ \\
\hline .0 & $788(57.94)$ & $820(60.29)$ & & $1001(57.53)$ & $907(52.12)$ & \\
\hline . 1 & $301(22.13)$ & $252(18.53)$ & & $384(22.07)$ & $419(24.08)$ & \\
\hline - $\geq 2$ & $271(19.93)$ & $288(21.18)$ & & $355(20.40)$ & $414(23.79)$ & \\
\hline Median Elixhauser comorbidity index (IQR) & $4(0-10)$ & $5(-4-9)$ & $<0.0001$ & $0(0-5)$ & $-2(-4-5)$ & $<0.0001$ \\
\hline Obstructive sleep apnea (\%) & $57(4.19)$ & $185(13.6)$ & $<0.0001$ & $90(5.17)$ & $304(17.47)$ & $<0.0001$ \\
\hline Tobacco use (\%) & $208(15.29)$ & $169(12.43)$ & 0.03 & $293(16.84)$ & $299(17.18)$ & 0.79 \\
\hline Alcohol use (\%) & $114(8.38)$ & $123(9.04)$ & 0.54 & $76(4.37)$ & $57(3.28)$ & 0.09 \\
\hline Procedure done as outpatient (\%) & $1017(74.78)$ & $1083(79.63)$ & $<0.01$ & $1591(91.44)$ & $1604(92.18)$ & 0.42 \\
\hline Endoscopist performing the procedure & & & $<0.001$ & & & $<0.0001$ \\
\hline Gastroenterologists & $857(63.01)$ & $762(56.03)$ & & $892(51.26)$ & $890(51.15)$ & \\
\hline Hepatologists & $503(36.99)$ & $598(43.97)$ & & $551(31.67)$ & $635(36.49)$ & \\
\hline Other providers & - & - & & $297(17.07)$ & $215(12.36)$ & \\
\hline
\end{tabular}

parable to each other. Data were stratified by EGD and colonoscopy and 1:1 match was conducted with propensity score margin being set at 0.1 . Logistic regression was performed separately for EGDs and colonoscopies to identify procedure related factors associated with obesity. Subgroup analysis was conducted by obesity class for EGDs and colonoscopies separately. The methodology and results of this analysis is provided in the Supplement file. All OR values were reported up to two decimal points except for values that would have rounded to 1.00. Twosided $P<0.05$ was considered significant. The analysis was performed with SAS software version 9.4 (SAS Institute Inc., North Carolina, United States). Additional details of statistical analysis can be found in the supplement file.

\section{Results}

\section{Data description and PSM}

A total of 7758 procedures were performed with moderate sedation during the study period. One or more variables were missing for one hundred and fifty-seven (2.02\%) procedures and these were excluded. Final analysis included 7601 procedures performed with moderate sedation for 5746 patients. Out of these 3545 (46.64\%) were EGDs and 4056 (53.36\%) were colonoscopies. Propensity score matching identified 1360 and 1740 pairs of EGDs and colonoscopies with moderate sedation for patients with and without obesity. - Table 1 summarizes the demographic and clinical variables and $>$ Table 2 summarizes the procedure related outcomes of patients undergoing EGD and colonoscopy.

\section{Comparison of obese and non-obese patients after PSM}

Median procedure time was shorter for obese patients (5 minutes, IQR 4 vs. 6 mins, IQR 5; $P<0.01$ ). For colonoscopies, cecal intubation time was slightly longer in obese patients (7 minutes, IQR 6 vs. 7 minutes, IQR 6; $P<0.01$; T Table 2). For EGDs, median administered dose of Midazolam ( $5 \mathrm{mg}$, IQR 3 vs. $5 \mathrm{mg}$, IQR 3; $P<0.01)$ and fentanyl $(100 \mathrm{mcg}$, IQR 50 vs. $75 \mathrm{mcg}, \mathrm{IQR}$ $50 ; P=0.01$ ) were higher for obese patients ( $\triangleright$ Table 2 ). Adjunctive sedatives were used more commonly in non-obese patients 
- Table 2 Procedure-related outcomes of patients with and without obesity undergoing EGD and colonoscopy with moderate sedation after propensity score matching.

\begin{tabular}{|c|c|c|c|c|c|c|}
\hline & \multicolumn{3}{|l|}{ EGD } & \multicolumn{3}{|l|}{ Colonoscopy } \\
\hline & $\begin{array}{l}\text { Non-obese } \\
(B M I<30 ; \\
n=1360 \text { ) }\end{array}$ & $\begin{array}{l}\text { Obese } \\
(B M I \geq 30 \\
n=1,360)\end{array}$ & $P$ value & $\begin{array}{l}\text { Non-obese } \\
(B M I<30 ; \\
n=1740)\end{array}$ & $\begin{array}{l}\text { Obese } \\
(B M I \geq 30 \\
n=1,740)\end{array}$ & $P$ value \\
\hline Median procedure time in minutes (IQR) & $6(4-9)$ & $5(4-8)$ & $<0.01$ & $21(16-28)$ & $21(16-29)$ & 0.75 \\
\hline Median Cecal intubation time in minutes (IQR) & - & - & - & $7(5-11)$ & $7(5-11)$ & $<0.01$ \\
\hline Median withdrawal time in minutes (IQR) & - & - & - & $12(9-18)$ & $13(9-18)$ & 0.41 \\
\hline Median recovery time in minutes (IQR) & $32(30-39)$ & $32(30-37)$ & 0.41 & $31(30-36)$ & $31(30-36)$ & 0.79 \\
\hline $\begin{array}{l}\text { Intra-procedure cardiopulmonary adverse } \\
\text { events (\%) }\end{array}$ & $\begin{array}{l}15 \\
(1.10)\end{array}$ & $\begin{array}{l}10 \\
(0.74)\end{array}$ & 0.32 & $\begin{array}{l}7 \\
(0.40)\end{array}$ & $\begin{array}{l}7 \\
(0.40)\end{array}$ & 1.00 \\
\hline 7-day all cause hospitalization (\%) & $\begin{array}{l}27 \\
(1.99)\end{array}$ & $\begin{array}{l}23 \\
(1.69)\end{array}$ & 0.57 & $\begin{array}{l}33 \\
(1.90)\end{array}$ & $\begin{array}{l}28 \\
(1.61)\end{array}$ & 0.52 \\
\hline $\begin{array}{l}\text { Poor tolerance of procedure or aborted } \\
\text { procedure (\%) }\end{array}$ & $\begin{array}{l}51 \\
(3.75)\end{array}$ & $\begin{array}{l}42 \\
(3.09)\end{array}$ & 0.34 & $\begin{array}{l}86 \\
(4.94)\end{array}$ & $\begin{array}{l}75 \\
(4.31)\end{array}$ & 0.37 \\
\hline Median dose of midazolam used in mg (IQR) & $5(4-7)$ & $5(4-7)$ & $<0.01$ & $5(4-7)$ & $5(4-7)$ & 0.24 \\
\hline Median dose of fentanyl used in mcg (IQR) & $75(50-100)$ & $100(50-100)$ & 0.01 & $100(75-125)$ & $100(75-125)$ & 0.86 \\
\hline Use of adjunctive sedation medications (\%) & $\begin{array}{l}191 \\
(14.04)\end{array}$ & $\begin{array}{l}155 \\
(11.4)\end{array}$ & 0.04 & $\begin{array}{l}174 \\
(10.00)\end{array}$ & $\begin{array}{l}167 \\
(9.60)\end{array}$ & 0.69 \\
\hline
\end{tabular}

undergoing EGDs (14.04\% vs. $11.4 \% ; P=0.04$; - Table 2 ). Other procedure-related outcomes for EGDs and colonoscopies were similar between the two groups ( $\triangleright$ Table 2 ).

\section{Regression model}

Recovery time was found to be shorter for obese patients undergoing EGD (OR: 0.989, 95\% Cl: 0.981-0.998; $P=0.01$ ). None of the other procedure related outcomes were different between patients with and without obesity for EGDs or colonoscopies.

\section{Per-patient sensitivity analysis}

Baseline population characteristics and regression model results from per-patient analysis for both EGDs and colonoscopies were similar to per-procedure analysis.

\section{Discussion}

This is the first study to evaluate procedure-related outcomes in obese patients undergoing endoscopy (EGD or colonoscopy) with moderate sedation. All procedure related outcomes for EGDs and colonoscopies performed with moderate sedation were similar between patients with and without obesity except for a shorter recovery time for obese patients undergoing EGD (OR: 0.99, - Table 2). However, the magnitude of this difference was so small that $O R$ had to be reported to 3 decimal points and the median recovery time for EGDs for both obese and non-obese patients was the same (32 minutes, > Table 2). This means that the statistical difference most likely happened from observations at the extremes ( $<25$ th or $>75$ th percentile) that happen far less frequently than observations clustered around the center (between 25th and 75th percentile). Our results are consistent with a prior study that reported no association of obesity with increase in procedure time and administered doses of fentanyl or midazolam [15].

Data on use of moderate sedation and their outcomes in obese patients undergoing endoscopy is limited to only a few studies that have focused on intra-procedure hypoxia or postprocedure patient comfort [16-18]. Obesity is known to alter the upper airway anatomy. Increased fat tissue in the head and neck areas predispose these patients to increased risk of obstructive sleep apnea (OSA), application of non-invasive and invasive ventilation devices during sedation $[19,20]$. This is compounded by a higher risk of impaired cardiopulmonary function and altered metabolism of sedative medications in obese patients [6-10]. OSA was found to be significantly more prevalent in patients with obesity in our population as well ( $\triangleright$ Table 1, - Table 3). All of these factors raise the theoretical concern of failure of moderate sedation or increased AEs and recovery time from moderate sedation in these patients. However, in our study no difference in sedative requirements was noted in obese patients compared to non-obese individuals. This was noted even for adjunct sedatives. Similarly, cardiopulmonary AEs and 7-day post-procedure hospitalizations were similar between obese and non-obese patients. Overall, our study suggests that moderate sedation can be used safely and effectively for performing endoscopic procedures in obese patients including patients with OSA, which is consistent with previous literature [21]. This is a critical finding especially because obesity 
- Table 3 Logistic regression model showing procedure related factors associated with obesity in patients undergoing EGD and colonoscopy with moderate sedation

\begin{tabular}{|l|c|c|}
\hline \multicolumn{1}{|l|}{ OR (95\% Cl) } & P value \\
\hline EGD & \\
\hline - Recovery time & $0.989(0.981-.998)$ & 0.01 \\
\hline
\end{tabular}

rate, endoscopy procedure volumes, its associated costs, and use of anesthesia for endoscopy procedures are all on the rise in the United States [1, 2, 22-24]. Judicious use of moderate sedation for certain endoscopic procedures in obese patients can help offset the burden on limited anesthesia resources and associated costs.

Unsedated procedures are an alternative option in patients deemed at high risk of sedation-related AEs. Two separate studies showed high success rate (92-98\%) of transnasal EGDs without sedation using an ultrathin scope in patients with morbid obesity $\left(\mathrm{BMI}>40 \mathrm{~kg} / \mathrm{m}^{2}\right)[25,26]$. The sample size in both studies was small (25 and 98), study populations were heterogenous (Veteran Affairs patients and patients in Brazil) and the EGDs were specifically done as a part of pre-bariatric surgery evaluation. Although, outcomes of unsedated colonoscopies have not been specifically studied in patients with obesity, colonoscopy without sedation has been performed with high success rate and patient satisfaction in selected patient groups $[27,28]$. Therefore, unsedated procedures can be considered in selected patients with obesity after a detailed risk and benefit discussion. Further studies are needed to specifically assess tolerance and satisfaction of unsedated procedures in patients with obesity.

This study has several strengths. The sample size was large $(n=7601)$ and complete data were obtained for $98 \%$ of procedures with only $2 \%$ of procedures having missing data. Propensity score matching was conducted to make the obese and nonobese populations as similar to each other as possible. A large number of variables were studied including multiple procedure-related outcomes (7 for EGDs and 8 for colonoscopies) and patient related factors ( 9 each for EGD and colonoscopy including prescription medications that can alter the effect of moderate sedation). None of the prior studies have looked at as many outcomes and patient variables. Additional analysis was performed for obesity classes to study the effect of increasing obesity on procedure related outcomes. Lastly, the AUC for regression models for EGD and colonoscopy were 0.63 and 0.69 , respectively. This is indicative of the robustness and reliability of the data analysis. In other words, the regression models accounted for $63 \%$ to $69 \%$ of the total variability in the data that can only be obtained with a large sample size and after accounting for major counfounders [29].

This study had some limitations as well. It was retrospective in nature that is associated with the inherent limitations of such studies. Reporting of patient intolerance in the procedure report by the endoscopist may have been limited. The intent of this study was to primarily to look at the type of intolerance that was severe enough to either abort the procedure, cause a cardiopulmonary AE or significant discomfort to the patient that necessitates use of anesthesia in the future. We were able to obtain information on that type of intolerance from the procedure reports. There was also a possibility of selection bias where endoscopic procedures for obese and morbidly obese patients with several comorbidities were performed with anesthesia and obese patients with fewer comorbidities had their procedures with moderate sedation. This limitation was addressed by performing PSM. Although PSM is not the same as randomization in a randomized controlled trial, this was the best statistical method available to control for known confounders. Finally, the diagnosis of OSA was based on chart review and could have been underdiagnosed in the study population. However, it was still found to be an independent predictor in the logistic regression models for both EGD and colonoscopy.

\section{Conclusions}

In conclusion, the results of this study strongly suggest that moderate sedation is well tolerated by obese patients undergoing EGD or colonoscopy without a clinically significant difference in most procedure related outcomes, AEs or post-procedure hospitalization. Further studies are needed to confirm these findings.

\section{Acknowledgements}

The project described was supported in part by the Translational Research Institute, grant UL1 TR003107 through the National Center for Advancing Translational Sciences of the National Institutes of Health (NIH). The content is solely the responsibility of the authors and does not necessarily represent the official views of the NIH.

\section{Competing interests}

The authors declare that they have no conflict of interest.

\section{References}

[1] Peery AF, Dellon ES, Lund J et al. Burden of gastrointestinal disease in the United States: 2012 update. Gastroenterology 2012; 143: 11791187

[2] Peery AF, Crockett SD, Murphy CC et al. Burden and Cost of Gastrointestinal, Liver, and Pancreatic Diseases in the United States: Update 2018. Gastroenterology 2019; 156: 254-272.e11

[3] Waring JP, Baron TH, Hlrota W et al. Guidelines for conscious sedation and monitoring during gastrointestinal endoscopy. Gastrointest Endosc 2003; 58: 317-322

[4] Braunstein ED, Rosenberg R, Gress F et al. Development and validation of a clinical prediction score (the SCOPE score) to predict sedation outcomes in patients undergoing endoscopic procedures. Aliment Pharmacol Ther 2014; 40: 72-82

[5] Ben-Menachem T, Decker GA, Early DS et al. Adverse events of upper Gl endoscopy. Gastrointest Endosc 2012; 76: 707-718 
[6] Jirapinyo P, Thompson CC. Sedation challenges: obesity and sleep apnea. Gastrointest Endosc Clin N Am 2016; 26: 527-537

[7] Alpert MA, Lavie C], Agrawal H et al. Cardiac effects of obesity: Pathophysiologic, clinical, and prognostic consequences-A review. J Cardiopulm Rehabil Prev 2016; 36: 1-11

[8] Alpert MA, Omran J, Bostick BP. Effects of obesity on cardiovascular hemodynamics, cardiac morphology, and ventricular function. Curr Obes Rep 2016; 5: 424-434

[9] Littleton SW, Tulaimat A. The effects of obesity on lung volumes and oxygenation. Respir Med 2017; 124: 15-20

[10] Dixon AE, Peters U. The effect of obesity on lung function. Expert Rev Respir Med 2018; 12: 755-767

[11] Van Walraven C, Austin PC, Jennings A et al. A modification of the elixhauser comorbidity measures into a point system for hospital death using administrative data. Med Care 2009; 47: 626-633

[12] Ladha KS, Zhao K, Quraishi SA et al. The Deyo-Charlson and Elixhauser-van Walraven Comorbidity Indices as predictors of mortality in critically ill patients. BMJ Open 2015; 5: 8990

[13] Thompson NR, Fan Y, Dalton JE et al. A new elixhauser-based comorbidity summary measure to predict in-hospital mortality. Med Care 2015; 53: 374-379

[14] Syed S, Baghal A, Prior F et al. Toolkit to compute time-based elixhauser comorbidity indices and extension to common data models. Healthc Inform Res 2020; 26: 193-200

[15] Czwornog J, Austin GL. Body mass index, age, and gender affect prep quality, sedation use, and procedure time during screening colonoscopy. Dig Dis Sci 2013; 58: 3127-3133

[16] Dhariwal A, Plevris JN, Lo NTC et al. Age, anemia, and obesity-associated oxygen desaturation during upper gastrointestinal endoscopy. Gastrointest Endosc 1992; 38: 684-688

[17] Madan AK, Tichansky DS, Isom J et al. Monitored anesthesia care with propofol versus surgeon-monitored sedation with benzodiazepines and narcotics for preoperative endoscopy in the morbidly obese. Obes Surg 2008; 18: 545-548

[18] Qadeer MA, Rocio Lopez A, Dumot JA et al. Risk factors for hypoxemia during ambulatory gastrointestinal endoscopy in ASA I-II patients. Dig Dis Sci 2009; 54: 1035-1040
[19] Mallampati SR, Gatt SP, Gugino LD et al. A clinical sign to predict difficult tracheal intubation; a prospective study. Can Anaesth Soc J 1985; 32: 429-434

[20] Juvin P, Lavaut E, Dupont $\mathrm{H}$ et al. Difficult tracheal intubation is more common in obese than in lean patients. Anesth Analg 2003; 97: 595600

[21] Andrade CM, Patel B, Gill J et al. Safety of gastrointestinal endoscopy with conscious sedation in patients with and without obstructive sleep apnea. J Clin Gastroenterol 2016; 50: 198-201

[22] Wang Y, Beydoun MA. The obesity epidemic in the United States Gender, age, socioeconomic, racial/ethnic, and geographic characteristics: A systematic review and meta-regression analysis. Epidemiol Rev 2007; 29: 6-28

[23] Travis AC, Pievsky D, Saltzman JR. Endoscopy in the elderly. Am J Gastroenterol 2012; 107: 1495-1501

[24] Inadomi JM, Gunnarsson CL, Rizzo JA et al. Projected increased growth rate of anesthesia professionaldelivered sedation for colonoscopy and EGD in the United States: 2009 to 2015. Gastrointest Endosc 2010; 72: 580-586

[25] Alami RS, Schuster R, Friedland S et al. Transnasal small-caliber esophagogastroduodenoscopy for preoperative evaluation of the highrisk morbidly obese patient. Surg Endosc Other Interv Tech 2007; 21: 758-760

[26] Xavier AT, Alvares AV, lyer PG et al. Unsedated transnasal endoscopy for preoperative examination of bariatric patients: a prospective study. Obes Surg 2020; 30: 238-243

[27] Petrini JL, Egan JV, Hahn WV. Unsedated colonoscopy: patient characteristics and satisfaction in a community-based endoscopy unit. Gastrointest Endosc 2009; 69: 567-572

[28] Aljebreen AM, Almadi MA, Leung FW. Sedated vs unsedated colonoscopy: A prospective study. World J Gastroenterol 2014; 20: 51135118

[29] Obuchowski NA, Bullen JA. Receiver operating characteristic (ROC) curves: Review of methods with applications in diagnostic medicine. Phys Med Biol 2018: 63 